\section{Stressinkontinenz: präoperative Urodynamik unnötig}

Patientinnen, deren Stressinkontinenz operativ behandelt wird, werden vor dem Eingriff urodynamisch untersucht. US-amerikanische Wissenschaftler stellen diese Praxis nun infrage, denn die Befunde haben ihrer Studie zufolge keinen Einfluss auf den Operationserfolg.

B ereits in der Vergangenheit hatten eine Cochrane-Analyse und das britische National Institute for Health and Clinical Excellence (NICE) den Nutzen der Urodynamik für den Operationserfolg angezweifelt, da sich die Indikation zur Operation vor allem am Leidensdruck der Inkontinenzpatientinnen orientiert. Nun liegen die Ergebnisse einer kontrollierten Studie vor.

An der Value of Urodynamic Evaluation (VALUE)-Studie beteiligten sich elf Zentren mit insgesamt 630 Patientinnen. Die Frauen litten seit mindestens drei Monaten an einer unkomplizierten Stressinkontinenz und hatten eingewilligt, sich einer Operation zu unterziehen. Waren diese Voraussetzungen erfüllt, wurden sie randomisiert entweder vor der Operation urodynamisch untersucht oder nicht. Als primären Endpunkt definierten die $\mathrm{Au}$ - toren den Operationserfolg nach zwölf Monaten. Als erfolgreich galt eine Operation, wenn die Punktzahl im Urogenital Distress Inventory, ein Fragebogen zum Ausmaß der Beschwerden, um mindestens $70 \%$ gesunken war, und wenn die Patienten die Symptomatik auf der Clinical Global Impression Improvement Skala (CGI-I) als „sehr viel besser“ oder „viel besser" bewerteten.

Die beiden Gruppen unterschieden sich in diesem Punkt nicht. Bei 76,9\% der Frauen mit urodynamischer Diagnostik und bei 77,2\% der Patientinnen ohne eine solche Funktionsanalyse war der Eingriff erfolgreich. Der Unterschied von $0,3 \%$ war statistisch nicht signifikant. Auch in den sekundären Endpunkten, wie Inkontinenzgrad, Lebensqualität, Patientenzufriedenheit, Anteil der positiven Provokationsstresstests, Blasenent- leerungsstörungen und Operationskomplikationen schnitten die beiden Gruppen ähnlich ab.

Zwar wurde bei Frauen mit urodynamischer Diagnostik seltener eine überaktive Blase und häufiger eine Blasenentleerungsstörung diagnostiziert als bei Frauen ohne weiterführende Diagnostik, doch das beeinflusste nicht die Wahl der Operationsmethode. Fast $93 \%$ der Frauen beider Studiengruppen wurden mit einer spannungsfreien Urethraschlinge versorgt.

Fazit: Der Operationserfolg unterscheidet sich bei Patientinnen mit Stressinkontinenz nicht - unabhängig davon, ob sie präoperativ urodynamisch untersucht werden oder nicht. Die Studienautoren sehen somit keinen Sinn in der routinemäßigen präoperativen Urodynamik.

Dr. Dagmar Kraus

Nager CW et al. A Randomized Trial of Urodynamic Testing before StressIncontinence Surgery. NEJM 2012; 366: 1987-97

\section{Akute Pyelonephritis: Sieben Tage Antibiose genügen}

\section{Bei Frauen mit akuter unkomplizierter Pyelonephritis ist eine siebentägige Ciprofloxacin-Therapie ausreichend. Die Erfolgsraten waren in einer randomisierten schwedischen Studie ähnlich hoch wie bei einer 14-tägigen Behandlung.}

U m die Resistenzlage zu verbessern, sollte der Antibiotikaverbrauch so weit wie möglich eingeschränkt werden. In einer prospektiven, randomisierten, doppelblinden Studie an 21 schwedischen Zentren wurde der Erfolg einer siebenmit einer vierzehntägigen Ciprofloxacintherpie bei insgesamt 156 Frauen (mittleres Alter 46 bzw. 41 Jahre) mit akuter Pyelonephritis verglichen.

In $92 \%$ der Urinkulturen wurde Escherichia coli als Infektionsverursacher nachgewiesen. Bei $27 \%$ der Frauen, überwiegend bei den älteren, war auch die Blutkultur positiv. Mit einer Kurzzeittherapie
(500 mg Ciprofloxacin $2 \times$ täglich) über sieben Tage konnte die Infektion bei $97 \%$ der Frauen ausgeheilt werden. Wurden die Frauen 14 Tage lang behandelt, lag die Erfolgsquote bei $96 \%$. Ob die Blutkultur zuvor positiv gewesen war oder ob ein schwerer Verlauf vorlag, spielte für das Ergebnis keine Rolle. Der kumulative Langzeiteffekt nach 28 beziehungsweise 42-63 Tagen erreichte in jeder der beiden Gruppen $93 \%$.

In beiden Therapiearmen wurde die Behandlung gut vertragen, dennoch ergab sich ein wesentlicher Unterschied: Während sich unter der Kurzzeittherapie bei keiner der Patientinnen eine Schleimhautmykose einstellte, war diese bei fünf Frauen der 14-Tage-Gruppe zu beobachten.

Fazit: Bei Frauen mit akuter Pyelonephritis ist eine siebentägige CiprofloxacinTherapie ausreichend. Die Autoren warnen jedoch, dass die hohe Heilungsrate, die unter einer Kurztherapie mit Ciprofloxacin gesehen wurde, nicht auf andere Antibiotikaklassen übertragen werden könne. Auch könne mit dieser Studie nicht sichergestellt werden, dass Patienten mit komplizierteren Infektionsverläufen die gleichen Erfolge hätten. Dr. Christine Starostzik

Sandberg $T$ et al. Ciprofloxacin for 7 days versus 14 days in women with acute pyelonephritis: a randomised, open-label and double-blind, placebo-controlled, non-inferiority trial. Lancet 2012; 380: 484-90 